Referencia para citar este artículo: Alvarez-Prieto, N. (2017). La violencia escolar en perspectiva histórica. Buenos aires, 1969-2010. Revista Latinoamericana de Ciencias Sociales, Niñez y Juventud, 15 (2), pp. 979-990. DOI:10.11600/1692715x.1521203082016

\title{
La violencia escolar en perspectiva histórica. Buenos aires, 1969-2010*
}

\author{
Natalia AlVAREZ-PRIETo** \\ Becaria Postdoctoral del Consejo Nacional de Investigaciones Científicas y Técnicas, Argentina.
}

\begin{abstract}
Artículo recibido en agosto 3 de 2016; artículo aceptado en febrero 2 de 2017 (Eds.)
\end{abstract}
- Resumen (descriptivo): desde hace más de una década, la violencia escolar ocupa un lugar importante entre las problemáticas investigadas en el campo de la educación. En este artículo, se presentan algunos de los principales hallazgos de una investigación cuyo objetivo general consistió en analizar el fenómeno desde una perspectiva histórica. El estudio fue realizado en tres escuelas públicas de nivel medio de la provincia de Buenos Aires (Argentina). Se emplearon como fuentes de información los documentos disponibles en los archivos de las escuelas seleccionadas, correspondientes al período 1969-2010. El análisis permitió comprobar que la violencia escolar no fue un problema de peso sino hasta la década del noventa. A su vez, se observó su íntima asociación con procesos más amplios de deterioro de la vida social.

Palabras clave: educación secundaria, violencia, investigación histórica, Argentina, (Tesauro de Ciencias Sociales de la Unesco).

\section{A historical perspective on school violence. Buenos Aires, 1969-2010}

- Abstract (descriptive): for over a decade, school violence has occupied an important place in educational research. This article presents some of the main findings of a study that had the overall objective of analyzing the phenomenon of school violence from a historical perspective. This study examined three public high schools in Buenos Aires (Argentina). Documents available in the schools' archives from the period 1969-2010 were used as sources of information. The analysis concludes that school violence wasn't an important problem until the nineties. The association of school violence with a general deterioration of social relations was also observed.

Key words: secondary education, violence, historical research, Argentina (Social Sciences Unesco Tesaurus).

\section{A violência escolar em perspectiva histórica. Buenos Aires, 1969-2010}

- Resumo (descritivo): há mais de uma década, a violência nas escolas ocupa um lugar importante entre as questões investigadas no campo da educação. Neste artigo, são apresentados alguns dos principais resultados de uma investigação cujo objetivo geral foi analisar o fenômeno a partir de uma perspectiva histórica. O estudo foi realizado em três escolas de ensino médio públicas

Este artículo de investigación científica y tecnológica se deriva de un estudio mayor titulado "La violencia escolar en perspectiva histórica. Un análisis de la trayectoria del fenómeno en escuelas públicas de nivel medio (Ciudad de Buenos Aires y Conurbano Bonaerense), 1969-2010", presentado por la autora para optar por el título de Doctora en Educación, Universidad de Buenos Aires, el 30 de marzo de 2016 (la investigación inició el 1 de abril de 2011 y finalizó el 31 de marzo de 2016), financiado por el Consejo Nacional de Investigaciones Científicas y Técnicas, resoluciones $\mathrm{n}^{\circ} 451$ del 10 de febrero de 2010 y n $^{\circ} 4773$ del 13 de diciembre de 2013. Estudio de corte histórico. Área de conocimiento: Ciencias de la Educación. Sub-área de conocimiento: Educación general.

** Licenciada en Sociología y Doctora en Educación de la Universidad de Buenos Aires. Becaria Postdoctoral del Consejo Nacional de Investigaciones Científicas y Técnicas. Orcid: 0000-0002-8208-5276. Índice H5: 2.Correo electrónico: nataliaalvarezprieto@gmail.com 
na província de Buenos Aires (Argentina). Foram usados, como fontes de informação, documentos disponíveis nos arquivos das escolas selecionadas, para o período 1969-2010. A análise constatou que a violência escolar não era um questão importante antes dos anos noventa. Observou-se também sua estreita associação com processos mais amplos de deterioração da vida social.

Palavras-chave: educação secundária, violência, pesquisa histórica, Argentina (Thesaurus de Ciências Sociais da Unesco).

\section{-1. Introducción. -2. Metodología de estudio. -3. Resultados. -4. Conclusiones -Lista de referencias.}

\section{Introducción}

Desde hace más de una década, la violencia escolar ocupa un lugar destacado entre las problemáticas estudiadas en el campo de la educación. El tema es también objeto de intervención desde las políticas educativas. De hecho, a partir de fines de los años noventa, distintos países crearon organismos específicos sobre la temática. El Observatorio Europeo de la Violencia Escolar ha sido pionero en ese sentido. Fundado en 1998 con el objetivo de investigar la problemática y elaborar propuestas de acción, en la actualidad, el organismo promueve acuerdos de cooperación entre distintos países del continente y otros extranjeros (Francia, Suiza, Polonia, Brasil, Canadá, México, Argentina). En Argentina, el Observatorio sobre la Violencia en las Escuelas fue creado en el año 2004. Asimismo, el Estado fue diseñando distintos programas educativos orientados a mejorar la convivencia en las aulas. Recientemente, incluso, la Legislatura sancionó una ley nacional para el tratamiento de la conflictividad social en las escuelas (Ley $\mathrm{n}^{\mathrm{o}}$ 26.892, Congreso de la Nación Argentina, 2013). Poco tiempo después, el Consejo Federal de Educación elaboró, además, una guía federal de orientaciones para la intervención educativa frente a situaciones de violencia (Res. $n^{\circ} 217$, 2014).

En el campo científico, también hacia fines de la década del noventa comenzaría a prestarse una mayor atención sobre el tema. En ese contexto, Tenti-Fanfani (1999) señalaba que las manifestaciones clásicas de la indisciplina escolar estaban siendo reemplazadas por nuevas formas de "desorden". Según el autor, la violencia presente en distintas áreas de la sociedad comenzaba a presentarse también en las escuelas. Pocos años después, en un informe para la Unesco, Filmus (2003) afirmaba que el incremento de la pobreza durante la década previa se expresaba en un crecimiento de los niveles sociales de violencia. Las escuelas eran convocadas a incluir a aquellos grupos excluidos de las demás esferas sociales. No extrañaba, entonces, la aparición en su seno de los primeros casos graves de violencia.

Desde aquel momento, se desarrollaron numerosas investigaciones sobre el fenómeno. En términos generales, el objetivo de esos estudios ha sido dilucidar las configuraciones culturales y las representaciones subjetivas sobre la violencia (Bustos-Raggi, OrtizMallegas, Pavez-Mena, Smith-Quezada \& Valdivia-Hennig, 2016; Kaplan \& Szapu, 2016; Langer \& Nievas, 2016; Morales-Tovar \& Silva-Castro, 2016; Di Napoli, 2015; HidalgoRasmussen \& Hidalgo-San Martín, 2015; Kaplan \& Di Napoli, 2015; Tomasini, 2015; Mutchinick, 2014; Observatorio Argentino sobre la Violencia en las Escuelas, 2013). Al examinar el estado del arte, se observó, en cambio, un importante vacío de conocimiento en torno a las formas concretas de la violencia y su evolución histórica. De allí surgió el propósito general de esta investigación: dar cuenta de la violencia escolar desde una perspectiva histórica.

Antes de iniciar el análisis resulta necesario definir el concepto "violencia escolar". En la presente investigación, se entiende por violencia el ejercicio de una fuerza que produce un efecto destructivo sobre las relaciones sociales. Se emplea el concepto "violencia escolar" en alusión a la violencia que se ejerce sobre una relación social particular, a saber, la relación escolar. En ese sentido, el término incluye toda violencia que se asienta sobre una 
relación escolar, se produzca dentro o fuera de la escuela o del período de clase. ${ }^{1}$ Agrupa, a su vez, distintas formas y niveles de gravedad. En ese sentido, la violencia puede ser física, verbal, simbólica o psicológica y puede escalar desde un "empujón" hasta un asesinato.

En este artículo se analiza la violencia escolar durante el período 1969-2010 en tres escuelas secundarias públicas de la provincia de Buenos Aires, a partir del empleo de fuentes documentales. Dichas fuentes fueron halladas entre los archivos históricos de las escuelas seleccionadas, previa autorización del trabajo de campo por parte de las autoridades escolares. ${ }^{2}$ Se trabajó con diferentes tipos de documentos (libros de actas generales, reuniones de personal, actas de convivencia, recomendaciones de rectoría, etc.), rastreándose a través de ellos toda la información disponible sobre situaciones de violencia ocurridas durante el período bajo estudio. La información fue registrada fotográficamente $\mathrm{y}$, luego, clasificada en función de un conjunto de variables significativas (período histórico, tipo de violencia, protagonistas, historia del conflicto, formas de intervención institucional, etc.). Finalmente, se realizó un análisis de tipo cualitativo de cada una de esas variables.

\section{Metodología de estudio}

Como se indicó antes, el objetivo principal de esta investigación consistió en analizar la violencia escolar desde una perspectiva histórica, durante el período 1969-2010, en las escuelas secundarias argentinas. Aquí se presentan algunos de los resultados principales de ese trabajo, correspondientes al examen realizado en tres escuelas públicas de la provincia de Buenos Aires. ${ }^{3}$

El diseño metodológico de esta etapa de la investigación fue de tipo cualitativo. Se

1 El término comúnmente empleado "violencia en las escuelas" remite, en cambio, al espacio físico en el cual se sitúa la violencia.

2 El trabajo de campo fue realizado durante los años 2013-2015.

3 En otras etapas de la investigación, el fenómeno fue analizado en el conjunto del territorio nacional empleando fuentes periodísticas y documentos oficiales. También fue estudiado, en mayor profundidad, en Capital Federal a partir del examen de los archivos documentales de tres escuelas públicas. recurrió a fuentes documentales pertenecientes a los archivos históricos de las escuelas seleccionadas. Allí se encontraron distintos tipos de fuentes de información: libros de actas generales, reuniones de personal, actas de convivencia, recomendaciones de rectoría, actuaciones del equipo de orientación escolar, entre otras. Los documentos relevados variaron en función de los archivos que fueron preservados por las escuelas durante más de cuatro décadas. De hecho, buena parte de los documentos escolares del período fueron deteriorándose o desechándose a lo largo del tiempo. La información específica sobre situaciones de violencia escolar se encontraba dispersa entre todo ese material, por lo que el trabajo de campo exigió una intensa labor de búsqueda y selección de la información.

Las escuelas seleccionadas se encontraban entre las pocas de la provincia que contaban con archivos históricos. ${ }^{4}$ Dos de ellas correspondían al partido de Malvinas Argentinas ${ }^{5}$ y la tercera a Lugones. ${ }^{6}$ Las dos primeras presentaban características edilicias similares. Se encontraban ubicadas en edificios relativamente pequeños, deteriorados, y compartían su espacio con escuelas de nivel primario. Se situaban, a su vez, en zonas semi-urbanas, rodeadas de casas bajas, calles de tierra y terrenos baldíos. Presentaban, también, características comunes en relación a su matrícula. En general, sus alumnos y alumnas provenían de familias obreras sumamente empobrecidas, quienes se trasladaban a la escuela desde los barrios más cercanos. ${ }^{7}$ Cabe destacar que la localidad se encontraba entre aquellas que reunían los peores indicadores sociales de la provincia. Para el año 2001, un 20\% de los hogares contaba con Necesidades Básicas Insatisfechas (siendo

4 En toda la provincia de Buenos Aires, solo cuatro escuelas medias públicas participan del Programa Nacional de Archivos Escolares.

5 De aquí en más se las denominará "Norte” y "Sur", por su ubicación dentro de la localidad.

6 De aquí en más se la denominará "Normal", por ser esa su modalidad educativa. El nombre de la localidad es ficticio. Se preserva la ubicación real de la escuela ya que el lector podría identificarla. Téngase en cuenta que la información analizada aquí es estrictamente confidencial.

7 Hasta mediados de la década del ochenta, las escuelas seleccionadas contaban con una matrícula compuesta solo por alumnos varones. 
$12,9 \%$ la media provincial) y más de un 50\% registraba algún tipo de "privación material" (siendo 38\% el índice provincial) ${ }^{8}$.

Por su parte, el Normal era una de las escuelas secundarias públicas más importantes de la zona. Al finalizar la jornada durante el turno mañana, el mismo edificio era ocupado por una escuela de comercio. Se trataba de un edificio muy amplio, muy deteriorado con el paso del tiempo, ubicado en una zona céntrica de la localidad. A diferencia de las otras dos escuelas, a lo largo de toda su historia, el Normal concentró una gran cantidad de alumnos, llegando a contar con ocho cursos en los primeros años del nivel. En relación a las características de la localidad, Lugones presentaba también indicadores sociales muy bajos con respecto a la media provincial, si bien en menor medida que los casos anteriores: de acuerdo al censo de 2001, un 15\% de los hogares presentaba Necesidades Básicas Insatisfechas y más de un $40 \%$ sufría alguna "privación material"; en 2010 , solo un $42 \%$ recibía agua de red y un $35 \%$ poseía desagüe cloacal.

El relevamiento de las fuentes documentales fue realizado durante los años 2013 y 2015, previa autorización de las autoridades escolares. En las tres escuelas se realizó un "contrato" de confidencialidad de la información. El trabajo de campo consistió en encontrar, entre las fuentes documentales examinadas, información relativa a episodios de violencia escolar. Una vez hallada, esa información fue registrada fotográficamente y clasificada en función de un conjunto de variables significativas (año, protagonistas, tipo de hecho, antecedentes, formas de intervención institucional, etc.).

El análisis de la información fue realizado a medida que se desarrollaba el trabajo de campo. En primer lugar, se volcaron todos los datos obtenidos en una matriz a partir de la cual pudieron destacarse categorías recurrentes. La tarea analítica consistió, principalmente, en realizar una lectura comprensiva de cada una de ellas. Finalmente, los datos fueron presentados en forma secuenciada de acuerdo a las etapas históricas más generales que atravesó el país durante el período: la década del setenta, la

8 Se emplearon datos estadísticos elaborados por el Instituto Nacional de Estadísticas y Censos. dictadura militar, la reapertura democrática, los años noventa y la década post crisis de 2001. De ese modo, el objeto de estudio fue examinado en su movimiento histórico, observándose sus transformaciones al calor de los cambios sociales y políticos más generales que vivió la sociedad argentina durante el período.

\section{Resultados}

\subsection{Los años setenta y ochenta}

Durante las dos décadas comprendidas entre 1969 y 1989, la violencia no fue una problemática de peso en la vida de las escuelas estudiadas. La principal fuente de conflictos era, en cambio, la disciplina del alumnado. Entre los temas más recurrentes se encontraban el comportamiento de los estudiantes dentro de la escuela, la vestimenta, la evasión de horas de clase y la relación y el trato con el cuerpo docente. El mantenimiento del orden escolar era un problema fundamental para las autoridades y los/las docentes. En ese sentido, las planificaciones de las diferentes asignaturas se proponían cultivar el esmero, el orden y la disciplina. Todas ellas, aptitudes fundamentales para la formación moral de los alumnos y las alumnas.

Preocupado por la disciplina en el colegio, el Rector del Normal formulaba, hacia 1970, un diagnóstico según el cual observaba una falta de adaptación de los alumnos y las alumnas a las normas disciplinarias de la escuela, especialmente durante los primeros años del nivel. De allí surgía la necesidad de un seguimiento "personalizado" de sus trayectorias, con el objeto de orientarlos en el proceso formativo. A partir de ese diagnóstico, se decidía crear la figura del "Profesor Asesor". Los asesores eran elegidos por el Rector, una vez al año, entre los y las docentes de cada curso. Su función era asesorar a los alumnos y las alumnas, a los padres y a sus colegas, así como a la Rectoría del colegio. En relación a los estudiantes, debía guiarlos en el modo de desarrollar sus tareas y ayudarlos a afrontar sus dificultades. También, aconsejarlos, estimularlos, interesarlos y comprometerlos con habilidad a superarse. Junto con los padres, 
debía analizar la situación escolar de los alumnos y las alumnas y asesorarlos sobre la mejor forma de colaborar en la tarea docente, participándoles sus inquietudes y haciéndoles conocer las dificultades así como sus posibles soluciones. Con los y las demás docentes, debía establecer un diálogo conducente a una labor más efectiva como resultado de un trabajo en equipo.

El cargo de "Profesor Asesor" permanecería a lo largo de toda la etapa, siendo incluso reforzado a mediados de los años ochenta con la creación de la figura del "Profesor Consultor". Este último se encargaría del seguimiento específico de los estudiantes con problemas de aprendizaje y conducta.

A partir de 1983, la política de "democratización" del sistema educativo daba lugar a una serie de cambios en el régimen de disciplina. ${ }^{9}$ Durante la década, la Dirección de Educación Media difundía una serie de documentos en ese sentido, en los cuales cuestionaba el "autoritarismo" presente en las prácticas docentes. Uno de los aspectos reformulados eran las normas de vestimenta y de presentación personal del estudiantado. El uso de uniforme en las escuelas públicas quedaba así en el pasado, al igual que las exigencias referidas, por ejemplo, al largo del cabello.

En ese nuevo marco, el trato y la relación entre los y las docentes y los alumnos y las alumnas comenzaban a ser problematizados bajo un nuevo enfoque. $\mathrm{Si}$, hasta ese momento, en la relación alumno/a-docente, solo la conducta de los primeros era objeto de cuestionamientos, ahora se sumaban una serie de críticas a las prácticas de los segundos. En ese sentido, por ejemplo, en agosto de 1984, el Consejo Consultivo del Normal llamaba la atención acerca de la "falta de consideración y las agresiones sobre los alumnos" por parte del cuerpo de profesores. Los y las docentes eran llamados asumir un nuevo rol, desprovisto de "autoritarismo" y abierto a la participación del estudiantado en el proceso educativo.

"El docente será el coordinador activo, sin carácter autoritario que guiará

$9 \quad$ En diciembre de 1983 finalizaba la última dictadura militar en el país, vigente desde marzo de 1976.

Rev.latinoam.cienc.soc.niñez juv 15 (2): 979-990, 2017 http://revistalatinoamericanaumanizales.cinde.org.co DOI:10.11600/1692715x.1521203082016 el proceso creativo. (...) Evitará el estereotipo, tomando el tiempo indispensable para discutir, opinar, encontrar puntos de coincidencia, distribuir responsabilidades, en suma abrir el espacio de la transformación del objeto a través de la participación de los sujetos" (Libro de Reuniones del Departamento de Materias Estéticas del Normal, 31/03/89).

Sin embargo, los cambios generaban una serie de tensiones entre las prácticas escolares tradicionales y el proceso democratizador. Hacia 1986, por ejemplo, los y las docentes manifestaban sus quejas frente a lo que caracterizaban como una situación extendida de indisciplina en el colegio. En términos generales, proponían reforzar dos aspectos: el respeto a los símbolos patrios y las normas de "urbanidad". En relación al primer punto, se buscaba dar importancia al acto de izamiento de la bandera. En cuanto a las normas de "urbanidad", el objetivo era que se estableciera un trato "considerado" y respetuoso entre los miembros de la comunidad educativa. Específicamente en el caso de los estudiantes, se indicaba que debían recibir de pie a los y las docentes cuando ingresaban en el aula. Asimismo, no se les permitía mascar chicles en clase, correr en las galerías de la escuela ni "jugar de manos". En cuanto a la "higiene", se les exigía mantener las paredes y bancos sin inscripciones y el piso del aula libre de papeles. Como se ve, se buscaba reforzar de ese modo un conjunto de normas disciplinarias de antigua data.

\subsection{Los noventa}

A partir de los años noventa, los hechos de violencia comenzaron a presentarse cada vez con mayor recurrencia e intensidad. Una de las fuentes más comunes de conflictos eran las agresiones verbales entre alumnos/as (gritos, insultos, burlas, etc.). También se registraban con frecuencia quejas por parte de familiares a propósito de los "malos tratos" ejercidos por el cuerpo docente sobre el estudiantado. Asimismo, las peleas entre alumnos/as en las que se observaba alguna forma de violencia 
física eran cada vez más habituales, dentro y fuera de la escuela, sobre todo a partir de la segunda mitad de la década.

Distintas formas de violencia "material" se presentaban también en forma cotidiana. En ese sentido, la destrucción de bienes y útiles escolares (bancos, sillas, muebles, pizarrones, puertas, paredes, etc.) y el robo de pertenencias entre estudiantes daban lugar a numerosos conflictos. Otro problema frecuente eran los robos y ataques a alumnos/as en los alrededores de las escuelas por parte de delincuentes. En ese sentido, por ejemplo, en julio de 1996, un conjunto de padres del Normal se reunía con las autoridades del colegio, alarmados frente a las agresiones sufridas por sus hijos/as en las cercanías del establecimiento.

"En una reunión de padres, solicitada por problemas de robos $y$ ataques físicos a sus alumnos, el Sr. Jefe de Preceptores comunica que el personal de preceptoría rota de lunes a viernes en el portón de ingreso de la calle para hacer ingresar a los alumnos y además cuenta con un personal que rota en las inmediaciones del establecimiento para prever cualquier inconveniente que pueda tener el alumnado" (Cuaderno de Preceptoría del Normal, 02/07/96).

Otra forma de violencia grave, si bien excepcional, era la presencia de armas en la escuela. El primer caso ocurría en el Normal, en junio de 1996, cuando un alumno de $1^{\circ}$ año llevaba una navaja y le producía cortes en la mano a una compañera. Durante ese mismo año, tenían lugar por lo menos cuatro casos más de alumnos portando armas "blancas" o de fuego en el colegio. Frente al agravamiento de las diferentes formas de violencia, los directivos de la escuela encomendaban al cuerpo de preceptores que realizaran informes anuales sobre los casos de agresividad y violencia. En 1998, los preceptores comenzaban a realizar recorridas permanentes, durante el horario de clases, controlando todos los espacios de la escuela (especialmente el buffet y los baños). En agosto del año siguiente, las autoridades hacían circular un documento titulado "Incidentes con armas, violencia o presencia de drogas", con el propósito de preparar a los y las docentes en las acciones a seguir frente a esas situaciones de "emergencia". Al mismo tiempo, desplegaban un conjunto de estrategias institucionales, tales como la revalorización de la figura de los profesores tutores, la realización de jornadas pedagógicas alusivas a la violencia y una mayor presencia del gabinete psicopedagógico en el día a día escolar.

\subsection{La década pasada (2000-2010)}

Durante la última década del período, la violencia escalaría aún más en frecuencia e intensidad. En las escuelas Norte y Sur, situadas en contextos sociales extremadamente pauperizados, llegaría a niveles críticos. Allí, la violencia escolar se sobreimprimía a otras múltiples formas de violencia en el seno familiar y en el contexto barrial. La violencia atravesaba, de ese modo, los ámbitos más importantes de socialización de los y las estudiantes.

Las condiciones de vida de la población de ambas escuelas eran muy adversas. Como se señaló antes, la mayoría de los estudiantes pertenecían a las capas más pauperizadas de la clase obrera. Distintos indicadores sociales daban cuenta de esa situación: alumnos/as que comenzaban a trabajar en forma muy temprana, padres y madres desocupados o empleados en trabajos muy precarios (empleo doméstico, construcción, "changas", etc.), familias que subsistían a través de planes sociales otorgados por el Estado, casos de desnutrición y falta de vestimenta para asistir a la escuela, etc. En esos contextos, muchas familias se encontraban desarticuladas, registrándose situaciones de abandono parental y hermanos o abuelos a cargo.

Es importante destacar que allí donde las condiciones de vida de la población escolar eran más desfavorables (el caso de la escuela "Sur"), se registraban, al mismo tiempo, niveles muy superiores de violencia escolar.

\subsubsection{Las formas de la violencia}

\section{Violencia simbólica y material}

En las escuelas estudiadas, la violencia simbólica se encontraba presente en la cotidianeidad escolar. Las agresiones verbales 
entre alumnos/as $\mathrm{y}$, también, hacia los $\mathrm{y}$ las docentes, eran un problema corriente. En general, los insultos y las burlas más comunes eran referidas al aspecto físico de los y las estudiantes ("gordo/a", "feo/a", "anteojudo/a"), a su sexualidad ("maricón", "puto", "afeminado", etc.) o se dirigían contra familiares o amigos/as. Esas situaciones, a su vez, daban lugar muchas veces a episodios de violencia física.

Las agresiones verbales contra un alumno o alumna en particular, expresadas en forma cotidiana, daban lugar muchas veces a situaciones de hostigamiento sostenido a lo largo del tiempo. ${ }^{10}$

"En el aula de $8^{\circ}$ en hora de Ciencias Sociales se llama la atención, reiteradas veces, al alumno M. porque molesta, verbalmente o por medio de dibujos, a su compañero A., refiriéndose a él como un afeminado. La profesora le llamó la atención en varias oportunidades, tratando de que reflexione, pero $\mathrm{M}$. no pierde oportunidad de ofender a su compañero. A. manifiesta estar cansado y que no dejará pasar otra ocasión y tomará medidas" (Libro de Actas del $3^{\circ}$ ciclo de la escuela "Norte", 21/11/06).

La violencia verbal no solo tenía como protagonistas a los y las estudiantes. En algunas ocasiones, se observaban quejas por la violencia verbal ejercida por los y las docentes sobre el alumnado.

"En el día de la fecha se presenta la madre del alumno V. de $1^{\circ}$ a manifestar que su hijo le lleva quejas del profesor de Ciencias Sociales. El menor sostiene que el docente le dice 'nene, tengo unas ganas de agarrarte y golpearte con un cinto y golpea la mesa"' (Libro de Actas Generales, escuela "Sur", 16/11/09).

Por otra parte, la destrucción de objetos y el robo de pertenencias -dos formas de violencia "material"- eran también problemas muy recurrentes. En algunos casos observados en la escuela Sur, los robos se encontraban

10 En la actualidad, esas situaciones se engloban dentro del fenómeno llamado "bullying", definido como el acoso moral, psicológico y/o físico de un alumno o grupo sobre otro, de un modo sistemático. Para ampliar, véase: Olweus, 1998. vinculados al problema del delito en el contexto barrial. La comunidad educativa manifestaba periódicamente su preocupación al conocer que ciertos alumnos/as robaban y/o vendían drogas en la escuela y en el barrio.

"En el día de la fecha se presenta la mamá de C. y F. acompañada por la mamá de un niño que no pertenece a la escuela. La señora viene a decir que hoy, aproximadamente a las 11.45, cuando su hijo volvía de la escuela, cruzando la plaza lo interceptaron tres alumnos de la escuela a quienes conoce como 'Carlitos', 'Chiche' y 'el Colo' precedidos por otros muchachos que no puede decir si pertenecen a la escuela. Los mismos estaban armados con palos, lo enfrentaron al niño 'Tito' y le robaron el celular; le quisieron robar la mochila. Manifiesta su preocupación porque no es la primera vez que tiene conocimiento de estos hechos donde estos alumnos participan. La Sra. manifiesta su preocupación por la integridad de sus hijos y que sabe por dichos de éstos que esos chicos venden y consumen drogas aún dentro de la escuela" (Libro de Actas Generales, escuela "Sur", 01/11/10).

\section{Violencia física}

La violencia física entre alumnos/as se encontraba presente en la cotidianeidad escolar. Esas situaciones presentaban diferentes niveles de intensidad, desde un "empujón" o tirón de pelo hasta una lesión grave.

“(...)esteúltimo[alumno]hamanifestado en los últimos tiempos situaciones de agresión a sus compañeros, los docentes y preceptora se quejan que el alumno no responde a los llamados de atención que se le imparten, tanto que en la última situación agredió tan mal al compañero S. que le hizo sangrar la nariz y la boca" (Libro de Actas del $3^{\circ}$ ciclo de la escuela "Norte", 15/05/07).

"En el día de la fecha, luego de finalizado el primer recreo, asisto al alumno C. de $1^{\mathrm{o}}$, quien presentaba un sangrado en la boca ya que se le "salió" una muela al ser golpeado por el alumno S. del 
mismo curso. Se llama a su domicilio y se dialoga con su hermana, ya que su mamá no se encontraba por motivos laborales" (Libro de Actas de Padres del Normal, 12/05/09).

Al igual que en el caso de las agresiones verbales, la violencia física podía convertirse en un problema crítico al ser ejercida en forma cotidiana contra un mismo alumno o alumna. Esos contextos de hostigamiento llevaban, en algunos casos, a que el/la estudiante agredido/a dejara de asistir por un tiempo o en forma permanente a la escuela. Frente a esas situaciones, las escuelas se veían obligadas a organizar un sistema específico para que los alumnos y las alumnas no desertaran y realizaran, en cambio, las actividades escolares en sus hogares hasta tanto se resolvieran los conflictos.

"En el día de la fecha se entrevista a la progenitora y a M., quien tiene el ojo izquierdo inflamado y con un moretón. La mamá comenta que ya fue agredido por otros compañeros. Además, el año pasado un compañero (L.) lo golpeó y se le cayó un diente. Fue atendido en el Polo Sanitario, además el año pasado le robaron en el curso el celular. La mamá dice que en muchas oportunidades, los compañeros de curso querían agredirlo por lo que él lleva el celular, por seguridad. (...) M. cuenta que L., desde principio de año lo molesta, y es molestado por sus compañeros, por lo que sus padres siempre informan estas situaciones a la Directora. Respecto al tema pedagógico, el día martes o miércoles se llevarán actividades para que M. las realice en su casa, hasta resolver el conflicto vincular y ver las acciones institucionales a seguir" (Libro de Actas del Equipo de Orientación Escolar, escuela "Sur", 25/09/09).

\section{Amenazas y presencia de armas}

Otra forma de violencia relativamente frecuente eran las amenazas entre estudiantes. En contextos sociales atravesados por la violencia, esas situaciones adquirían una dimensión mayor llevando, incluso, a los/as

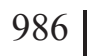

alumnos y alumnas a abandonar la escuela. En el caso de la escuela Sur, el temor entre la población escolar frente a las amenazas -las cuales, muchas veces, se materializaban en agresiones físicas- era un problema de primer orden ${ }^{11}$.

"Se recibe a la madre del alumno R., de $1^{\circ}$ año, para informar que su hijo no está asistiendo a la escuela por temor frente a amenazas, ya que el menor explica que otro alumno lo amenazó de que lo golpearía con otros chicos del barrio. Pregunto frente a los rumores de que hubo un arma en la escuela y dice que un día, hace aproximadamente dos semanas, B. le mostró un revólver. Se pregunta por qué razón no informó esto a un adulto y explica que por miedo. Averiguo además en qué contexto le mostró el arma y le dijo: "esta es para los de tu barrio y si vos te metés para vos también". La mamá manifiesta temor e intenciones de cambio de escuela" (Libro de Actas Generales, escuela "Sur", 18/05/10).

Frente a los casos más graves, las familias y las autoridades escolares debían desplegar una serie de medidas para garantizar la seguridad de los y las estudiantes. Algunas veces, por ejemplo, decidían acompañar a los alumnos y las alumnas en el trayecto entre sus hogares y la escuela. Una situación de ese tipo tenía lugar, por ejemplo, en la escuela Norte a raíz de las amenazas cotidianas -en la escuela y en el barrio- realizadas por un alumno contra sus compañeras mujeres. La situación generaba una profunda preocupación en la comunidad educativa en tanto se sospechaba que el joven llevaba armas a la escuela.

"La alumna G., cursando el tercer ciclo de adultos, le cuenta a la profesora que había sido amenazada por N. con un arma si ella no le entregaba una carta a quien fue su novia el año pasado. También lo vieron en el salón de clase armando "porros"12 y le dijo a G. que

\footnotetext{
11 En la escuela, además de registrarse numerosos casos de abandono escolar, se observaba una alta rotación del cuerpo docente y directivo.

12 Cigarrillos de marihuana.
} 
se callara la boca porque si no le iba a meter un tiro. G. nos informa que ayer volvió a amenazarla a ella y a la mamá y también dijo que anda armado por si se llega a cruzar con alguno de los hermanos de su ex novia. La mamá fue el día de ayer a hacer la denuncia a la comisaría pero no se la quisieron tomar porque tienen miedo a que N. tome represalias" (Libro de Actas del $3^{\circ}$ ciclo de la escuela "Norte", 31/03/06).

Una forma grave de violencia que se presentaba ahora con mayor frecuencia, respecto a la década anterior, era la presencia de armas en la escuela. Durante el período, en las tres instituciones estudiadas se conocían casos de alumnos armados. Llevar un arma a la escuela podía tener distintos significados, desde exhibirla por curiosidad o diversión hasta amenazar a un/a compañero/a.

El problema era mucho más frecuente y adquiría niveles mayores en la escuela Sur. Debido a ello, en distintas ocasiones la comunidad educativa manifestaba su preocupación por la seguridad de los alumnos y las alumnas.

"La Sra. Directora informa que recibirá a los papás interesados en dialogar con ella para plantear sus inquietudes, a las que, en conjunto buscarán una respuesta frente a la denuncia concreta que efectuaron sobre la presunción de irrupción de algunos alumnos que pudieran traer algún tipo de arma. La Dirección ofrece la tranquilidad a los padres que frente a la situación concreta se dará inmediatamente intervención policial" (Libro de Actas Generales, escuela "Sur", 17/05/10).

Al igual que en el caso de las amenazas, la presencia de armas en el ámbito escolar se vinculaba muchas veces al problema del delito y la inseguridad en el barrio. ${ }^{13}$ Los alumnos

13 Las agresiones por parte de patotas barriales impactaban muchas veces en la asistencia de los estudiantes a la escuela. En el caso de la escuela Sur, se registraban incluso algunos casos de abandono escolar por ese motivo: "En el día de la fecha se presenta la abuela del alumno V. de $2^{\circ} \mathrm{C}$ quien fuera citada ya que su nieto no viene a la escuela. Explico que el alumno debe regresar a la escuela para poder continuar sus estudios. La Sra. manifiesta que teme por la seguridad de su nieto que tiene un problema barrial donde adultos lo tienen amenazado. Pregunto si realizó y las alumnas eran usualmente víctimas de agresiones por parte de delincuentes en las cercanías de la escuela. En ese contexto cargado de peligro, algunos de ellos decidían llevar un arma consigo como una forma de "autodefensa". A la inversa, también lo hacían aquellos/as alumnos/as que formaban parte de ese mundo delictivo.

\subsubsection{Los protagonistas}

La mayoría de los hechos de violencia escolar analizados antes tuvieron como protagonistas a alumnos y alumnas, ya sea como agresores o como víctimas. Si bien en menor medida, también se registraron algunos casos protagonizados por docentes. Un fenómeno particular observado en las escuelas Norte y Sur era la intervención de familiares y amigos en las disputas entre alumnos/as. En esos casos, al extenderse a otros ámbitos de socialización, los enfrentamientos escolares se agudizaban aún más. De ese modo, resultaba muy difícil resolverlos dentro del marco institucional de la escuela.

Algunas de esas situaciones adquirían niveles preocupantes, llevando a las autoridades y a los y las docentes a adoptar una serie de medidas ad hoc para proteger a los y las estudiantes.

"La profesora avisa al personal policial que pasa regularmente a firmar la asistencia, que S. amenazó a E. y que lo iban a agarrar con sus amigos a la salida. El personal policial expresa que estaba ocupado, pero iba a tratar de dispersarlos. (...) A las 17.05 horas los alumnos salen, S. acompañado por su grupo de amigos comienza a provocarlo, ante esta situación el profesor de Matemáticas se acerca al grupo para dialogar, pero no se logra ningún resultado positivo. Ante esta situación, la preceptora decide acompañar al alumno hasta la parada de colectivo, también lo hacen otros compañeros de E. Al llegar a este lugar,

la denuncia policial y la Sra. expone que no, que tiene miedo. Manifiesta que no consigue vacante en otra escuela" (Libro de Actas Generales, Escuela "Sur", 21/04/10). 
los amigos de S. siguen provocando al alumno de 9no, entonces éste dice que no tiene miedo y se van a las manos, resultando golpeado E. Se separa a los dos chicos. S. se va con sus amigos, advirtiendo que esta vez se salvó pero la próxima no. E. es llevado a la casa de la preceptora, desde allí se llama a su familia; no encontrándose los padres en su hogar" (Libro de Actas del $3^{\circ}$ ciclo de la escuela "Norte", 03/07/06).

Muchas veces, los episodios de violencia entre alumnos/as hundían sus raíces, en realidad, en disputas previas surgidas en el barrio. A la inversa, en algunas ocasiones, los enfrentamientos dentro del espacio escolar se trasladaban, luego, al ámbito barrial. Como se ve, la "frontera" entre el afuera y el adentro escolar resultaba muy difusa.

"En el día de ayer, aparentemente, E. habría pasado a la tarde por la casa de G. y otras chicas, gritando "hija de puta", aparentemente estaba también otro alumno de la escuela. El papá comenta que hace siete días pasó por la escuela e informó que había en la puerta de la escuela, siete adolescentes entre varones y mujeres, que estaban esperando a G., amenazando que le iban a pegar. Había una joven, dice el papá, que no sería de la escuela, con una navaja."

"En el día de la fecha se hace presente la hermana del alumno N., debido a una citación de Gabinete. La misma expone que sucedió un conflicto fuera de la escuela con el alumno C. En respuesta, la mamá de C. se dirigió a su domicilio con insultos y se enteran todos los vecinos. La hermana expresa que se encontraron con una citación policial por amenazas. El problema viene de años, según ella, el hermano hasta hoy no reaccionó violentamente, pide que no haya tratos con ellos."

Todas estas situaciones tenían lugar en contextos sociales conflictivos, atravesados por múltiples formas de violencia $\mathrm{y}$, en términos más generales, por procesos de descomposición de los vínculos sociales.

\subsubsection{El contexto social}

En las escuelas Norte y Sur, los elevados niveles de violencia escolar se correspondían con distintos indicadores de procesos más amplios de degradación personal y de descomposición de la vida social. ${ }^{14}$ En relación al primer punto, las adicciones y el consumo de sustancias psicoactivas eran un problema recurrente entre el alumnado. Asimismo, se registraban periódicamente situaciones que ponían de manifiesto el sufrimiento experimentado por los y las estudiantes: "ataques de ansiedad", síntomas de depresión, deseos de muerte, intentos de suicidio, etc. La presencia cotidiana de la violencia en el ámbito familiar y en el contexto barrial daba cuenta, por su parte, de la descomposición del vínculo social.

Como se vio antes, los conflictos familiares, barriales y escolares se entrecruzaban de diferentes formas. Ese entrecruzamiento configuraba contextos atravesados por la violencia en forma global. La presencia generalizada de la violencia se expresaba, a su vez, en las prácticas de los/las adolescentes de diferentes formas. Las más características eran el retraimiento, la agresividad en el vínculo con otros y las conductas autodestructivas o de riesgo ${ }^{15}$.

"En el día de la fecha se presentó la mamá de la alumna P. de $8^{\circ}$ B. La Sra. expresa que su hija tomó cuatro pastillas para dormir el fin de semana. La alumna cuenta que uno de los compañeros nuevos amenaza en el curso con que al rendir todas las materias iba a violar luego a todos los chicos. A partir de estos dichos y luego de profundizar en la entrevista, se deriva en la denuncia efectuada por P., porque termina informando que el almacenero del barrio intentó abusar sexualmente de ella. A partir de entonces, sus hábitos y carácter cambiaron. (...) El Orientador

14 Aquí se denomina "descomposición social" al quiebre del conjunto de relaciones que conforman y estructuran la realidad social.

15 Cabe señalar que, en la mayoría de los casos, los estudiantes víctimas de la violencia en el ámbito familiar presentaban problemas de aprendizaje y asistían a la escuela en forma intermitente. 
Social sugiere a la mamá que consulte en carácter de urgente al Asistente Social del Centro de Salud, solicitando atención al Hospital de Salud Mental, por intento de suicidio. Se sugiere al padre quien se incorpora a la reunión, que se calme y que realice la denuncia correspondiente" (Libro de Actas del Equipo de Orientación Escolar, escuela "Sur", 26/03/07).

La violencia en el seno familiar era un problema muy extendido, especialmente en la escuela Sur. Adquiría en algunos casos características muy graves. En el año 2008, por ejemplo, la Orientadora Social de la escuela acompañaba a la abuela de una alumna a realizar una denuncia contra los padres por maltrato físico y emocional. En el hospital de la zona, los médicos constataban las lesiones físicas que presentaban la joven y sus hermanos. En 2010, las autoridades de la escuela intervenían en el caso de un alumno que se producía cortes en sus brazos. Según los informes del Equipo de Orientación, él y sus hermanos sufrían privaciones materiales y eran víctimas de maltrato y abandono familiar. Frente a ese cuadro de situación, la escuela derivaba al alumno al servicio de salud mental para que fuera atendido en forma urgente. Ese mismo año, se conocía el caso de una alumna golpeada por la pareja de su madre. La mujer también era víctima de las agresiones y había llegado, incluso, a ser hospitalizada por los golpes recibidos. Frente a ese cuadro, la justicia le quitaba la patria potestad y enviaba a la joven a vivir con su padre biológico.

De lo anterior se desprende la necesidad de comprender la violencia escolar a la luz de las características más generales del contexto social en el que se ubica la escuela.

\section{Conclusiones}

En este artículo pudieron observarse dos cuestiones de importancia en relación a la violencia escolar durante el período estudiado. Por un lado, una tendencia al crecimiento y a la agudización del fenómeno en el largo plazo. Por el otro, su relación con procesos más amplios de quiebre del vínculo social. En ese sentido, se comprobó que allí donde las relaciones sociales se encontraban más deterioradas, la violencia escolar se presentaba con mayor frecuencia e intensidad.

En relación al primer punto, las diferentes formas de violencia fueron en aumento $y$ adquirieron niveles mayores con el paso del tiempo. No fue sino hasta los años noventa que la violencia escolar se convirtió en un problema de peso, especialmente a partir de mediados de la década. A partir de aquel momento, comenzaron a registrarse además algunas formas de violencia muy graves, tales como la presencia de armas en la escuela.

En relación al segundo punto, en los casos analizados, la violencia escolar se encontraba asociada a otras formas de violencia en el contexto familiar $y / 0$ barrial y a distintos indicadores de "descomposición" de la vida social. Se registraban, de ese modo, múltiples entrecruzamientos entre distintas formas de violencia. A su vez, la violencia social y la violencia escolar se encontraban mucho más extendidas y entrelazadas allí donde las condiciones de vida de la población eran más adversas.

De allí surge la necesidad de indagar con mayor detenimiento las lógicas de la violencia entre los sectores más afectados por los procesos de pauperización social. En una próxima etapa de esta investigación, se estudiarán las formas de producción de la violencia escolar entre estudiantes de nivel secundario en contextos sociales de clase obrera atravesados por procesos agudos de empobrecimiento social. Se observará de qué manera nacen y se desarrollan los hechos de violencia en esos contextos; el significado de los motivos desencadenantes y las configuraciones culturales que se encuentran detrás de ellos (por ejemplo, qué sentido tiene para los y las estudiantes estar en la escuela, qué valor e importancia les otorgan a los vínculos escolares, etc.). De esa manera, se obtendrá un nivel aún mayor de profundidad en el análisis del fenómeno.

\section{Lista de referencias}

Bustos-Raggi, C.; Ortiz-Mallegas, S.; PavezMena, J.; Smith-Quezada, D. \& Valdivia- 
Hennig, N.(2016). Concepcionesdeinfancia en una escuela con altos índices de violencia escolar de estudiantes a profesores. Revista Latinoamericana de Ciencias Sociales, Niñez y Juventud, 14 (2), pp. 1145-1159. Doi: 10.11600/1692715x.14218210814.

Congreso de la Nación Argentina (2013). Ley No. 26.892 para la promoción de la convivencia y el abordaje de la conflictividad social en las instituciones educativas. Buenos Aires: InfoLEG.

Consejo Federal de Educación (2014). Resolución No. 217 "Guía federal de orientaciones para la intervención educativa en situaciones complejas relacionadas con la vida escolar". Buenos Aires: Consejo Federal de Educación.

Di Napoli, P. (2015). La juventud como objeto de temor y estigmatización. Sentimientos desde y hacia los jóvenes de los países del Cono Sur. Revista de Ciencias Sociales, 29 (38), pp. 123-144.

Recuperado de: http://cienciassociales.edu. uy/departamentodesociologia/wp-content/ uploads/sites/3/2016/07/Articulo-Napoli. pdf

Filmus, D. (2003). Enfrentando la violencia en las escuelas: un informe de Argentina. En J. Werthein (ed.) Violência na Escola: América Latina e Caribe, (pp. 15-79). Brasilia: Unesco.

Hidalgo-Rasmussen, C. \& Hidalgo-San Martín, A. (2015). Violencia e inseguridad contextual percibida y roles en bullying en escolares mexicanos. Revista Latinoamericana de Ciencias Sociales, Niñez y Juventud, 13 (2), pp. 767-779. Doi: 10.11600/1692715x.13215021214.

Kaplan, C. \& Di Napoli, P. (2015). La dimensión de la emotividad en la educación secundaria: aportes y desafíos teóricos y empíricos en los estudios sobre violencia y subjetividad social. En D. Pinkasz (comp.) La investigación sobre educación secundaria en la Argentina en la última década, (pp. 125-138). Buenos Aires: Flacso.

Kaplan, C. \& Szapu, E. (2016). Marcas subjetivas. Racismo y discriminación en la trama educativa. Mídia: a produção do consenso e a cultura da violência. Ponencia presentada en el XI Simposio Científico Cultural, Paranaíba, Brasil.

Langer, E. \& Nievas, A. (2016). Las vivencias sobre la violencia de jóvenes en una escuela en contexto de pobreza urbana. Juventudes en disputa: permeabilidades y tensiones entre investigaciones y politicas. Ponencia presentada en la $\mathrm{V}$ Reunión Nacional de Investigadores/as de Juventudes Argentinas, Rosario, Argentina.

Morales-Tovar, L. \& Silva-Castro, D. (2016). Representaciones sociales de los estudiantes con relación a la violencia entre pares. Revista Aletheia, 8 (2), pp. 86103. Recuperado de:

http://aletheia.cinde.org.co/index.php/ ALETHEIA/article/view/323/226

Mutchinick, A. (2014). Las humillaciones entre estudiantes secundarios. Sentimientos $y$ consecuencias sobre la autoestima y la experiencia escolar. Sociedades contemporáneas, Subjetividad y Educación. Ponencia presentada en las II Jornadas Internacionales, Buenos Aires, Argentina.

Observatorio Argentino de Violencia en las Escuelas (2013). Sobre la violencia en las escuelas. Desde la mirada de los alumnos. Buenos Aires: Ministerio de Educación de la Nación.

Olweus, D. (1998). Conductas de acoso y amenaza entre escolares. Madrid: Morata.

Tenti-Fanfani, E. (1999). Más allá de las amonestaciones. Buenos Aires: Unicef.

Tomasini, M. (2015). Feminidades juveniles, cuerpos y producciones estéticas en la escuela secundaria. Revista Iberoamericana de Educación, 68, pp. 17-136. Recuperado de:

http://rieoei.org/rie_revista.php?nu mero $=$ rie 68 a $06 \&$ titulo $=$ Feminidad es $\% 20$ juveniles, $\% 20$ cuerpos $\% 20 \mathrm{y} \% 20$ producciones $\% 20$ est $\%$ C3\%A9ticas $\% 20$ en $\% 201 \mathrm{l} \% 20$ escuela $\% 20$ secundaria 